tion would be much less monstrous. Whilst a man's intellectual faculties may be calculated to give him a respectable place in the ranks as a rational being, by his moral faculties he may approach the level of the animals that have no reason. The picture may also be reversed; and these two extremes may meet through every gradation in the relative excellence of the duplicate powers.

Now we have seen that the intellectual faculties may undergo a change from health without very conspicuously implicating the moral powers in the disorder. I do not say that the intellect can be very largely destroyed by disease, and the moral powers of the mind left unimpairel; there is that amount of connection and sympathy between them which involves the one, to a greater or less extent, in any violent injury which may befall the other. But observation shows that the integrity of the intellect can be invaded by disease, whilst the moral powers remain possessed of a large share of soundness, and the residence of the latter faculties in a distinct division or region of the brain, would afford a satisfactory explanation of this phenomenon. Then let me ask, if this be so, can any reason be imagined why the moral powers themselves should not be liable to the invasion of disease, to an equal extent, without involving the intellectual powers? If we grant the localisation of the different orders of faculties in different regions of the brain, and allow that disease may have its principal seat in that part of the brain assigned to the intellectual faculties, what hinders us from the converse conclusion, that the chief seat of the disease may be in that region of the brain which performs the moral and instinctive functions? Nay, I think the inference is inevitable, that such may be the case, and I think further, that unprejudiced observation conclusively establishes the fact that such is the case. Here, then, we get to the rationale of moral insanity, to the very fons et origo of those moral, affective and instinctive perversions which show themselves in such a vast variety of forms, producing eccentricities of character of every conceivable and possible kind, evincing themselves in otherwise unaccountable modes, sometimes leading to unmistakable insanity, and sometimes to such manifestations of character as lay the foundation for legal investigation into the person's accountability, and at others merely occasioning the resemblance to an intractable, vicious, and ill-conditioned mind. Here, however, we have the explanation of all these difficulties. How simple it appears, when thus stated! but how complicated in its practical application. The infinite diversities in the moral character of different individuals, produced by the various degrees of force in the separate faculties relatively to each other, all acted upon by the singularly diversified agencies of outward circumstances, the improving or vitiating influences of education, and of subjective training, result in phenomena so complex, that the satisfactory application of the simplest doctrine is often extremely difficult. The difficulty is also further increased by the two conditions under which moral insanity is exhibited-either as it may be congenital, or as it may supervene in after life.

[To be continued.]

\section{Gunlstomian aldectures:}

\section{ox}

\section{DIABETES AND SACCHARINE CONDITIONS OF THE URINE.}

DELIVERED AT THE ROYAL COLLEGE OF PHYSICIANS, FEBRUARY 25TH, 27TH, AND MARCH 4TH, 1857.

By Alfred B. Garrod, M.D., Fellow of the Royal College of Physicians; Professor of Materia Medica, Therapeutics, and Clinical Medicine, at University College; and Physician to the Hospital.

\section{LECTURE III (continued).}

I HAVE been careful to give you the absolute numbers obtained in some of my experiments upon the influence of diet upon the quantity and quality of the urine in cases of diabetes, because I consider the subject one of great importance, especially in reference to the treatment of the disease; and although, I believe, at the present day most practitioners recommend an animal diet to their patients, yet I am sure that many are inclined not to lay sufficient stress upon it. Dr. Prout gives the following as his reasons for not adhering to such a plan chiefly founded, you will observe, upon an hypothesis as to the assimilation of saccharine principles which at present would scarcely be considered as well established. $\mathrm{He}$ remarks: "For some years past, a diet exclusively animal has been much insisted upon. I do not, however, approve of a diet exclusively animal; but consider a certain proportion of furinaceous matters proper. The recommendation of this admixture of farinaceous matters is founded upon a fact apparently well established; viz., that the assimilation of the saccharine principle is one of the last functions which becomes extinct in animals. The proportions of these two forms of aliment must be varied according to the circumstances of the patient, and particularly according to the degree in which he is able to assimilate albuminous, in preference to farinaceous matterspoint not difficult to be determined by a little attention."

Other physicians have supposed that the total abstraction of amylaceous matters may produce a shock on the patient's system.

In opposition to these views, I may remark, first, that patients are able to bear a strictly animal diet, without the production of any shock to the system, or injury to their health; secondly, that those who have had the greatest success in the treatment of these patients have most exclusively adhered to and strongly recommended such a plan; and lastly, that, as most diabetics, even when not partaking of amylaceous matters, still pass sugar in their urine, and as the amount of this substance, together with the diabetic symptoms in many cases, increases in the direct ratio with the quantity of such taken by the individuals, it follows that, in such patients, any amylaceous or saccharine matters in the food do not become assimilated, but act only as irritants, and should therefore be avoided as far as possible.

I have said that, in many cases, the sugar in the urine bears a strict relation to the amount of amylaceous matters in the food. This does not hold good in some, or at least in certain stages of the disease; and I am of opinion that the power of assimilating or converting such principles into fatty or other substances, which $I$ have regarded as existing in the healthy liver, but injured or destroyed in diabetes, may be restored, and still the urine may remain saccharine under a rigid animal diet, from the persistence of the over sugar-forming function of the organ. I could bring forward examples illustrating this point, which is one of very considerable importance in the treatment of the disease, as by it we may be safely guided as to the propriety of allowing a return to the use of bread and vegetables.

Then comes the difficulty, How can a strict diet be devised? After a very short time, patients become disgusted with meat, eggs, etc., unless there is some admixture of bread or vegetable matter. Again, it is desirable to have substances more bulky, and containing less nutritive matter than meat, in order to satisfy the appetite; and this applies especially to a disease in which the cravings of hunger are often very great. Many suggestions have been made. M. Bouchardat proposed, some year's since, the use of a species of bread called pain de gluten, or gluten bread, which is made by washing flour in order to deprive it of the chief portion of the starch, and then forming it into bread or cakes. As made in this country, as far as my experience goes, it is not very palatable, and often contains a very considerable proportion of fecula; and, lastly, it is very costly-a most important item in an article of daily consumption. Nany, I find, would much rather simply limit considerably the quantity of amylaceous matters, than take this gluten bread. I have met with some practitioners who have entertained the idea that gluten has peculiar virtues in this disease, and, therefore, that the advantage would be in proportion to the amount taken; but of course this is erroneous, and the pain de gluten is of use only in proportion as it enables the patient to abstain from amylaceous matters. In Paris, gluten is prepared in several forms; much more attention is paid to its manufacture, and a very superior article is produced-one, in fact, by no means unpalatable.

Many other substitutes have been proposed; all, however, with the exception of the one of which I will now speak, have been received with little or no favour, and have either scarcely been employed at all, or have soon fallen into disuse. This substitute for bread-by far the best with which I am acquainted-is made from bran, and has been brought under the notice of the profession by Mr. Camplin, a medical gentleman of this metropolis, who has himself been a sufferer from diabetes for more than twelve years, but who, I am pleased to add, has been enabled to keep himself in good health, and able to undergo the fatigues of an arduous profession to the present time; and this has been due, in his opinion (in which I would fully coincide), to his being able to keep to a rigid diet, during the time that symptoms have required it, by the use of this 
article of food. Mr. Camplin, in his paper published in the thirty-eighth volume of the Medico-Chirurgical Transactions, and entitled "On the Juvantia and Lædentia of Diabetes," which I would recommend strongly to your perusal, as containing many valuable hints and experiences, states that the first suggestion was due to Dr. Prout, but that, when made according to the directions there given, it could not be continued, chiefly on account of the irritation which it produced on the bowels. Mr. Camplin has been able to overcome this difficulty by reducing the bran to a very fine powder by means of a mill specially adapted for the purpose. The mode of making his cake or bread is as follows :-Bran, after being washed thoroughly, to free it from any adhering flour, is dried, and reduced to an impalpable powder; it is then made into cakes by means of butter, eggs, and milk, and baked in little pans. For the details of the process I must refer you to his paper. Recently Mr. Camplin has somewhat altered his formula, and, in place of forming cakes, causes the evolution of carbonic acid in the mass by means of carbonate of soda and hydrochloric acil, and thus a light form of bran bread is produced. Through the kinclness of Mr. Camplin, I am now enabled to exhibit this bread, and also the bran reduced to its proper degree of fineness.

Dr. Prout was inclined to think that amylaceous or saccharine substances were not all equally injurious; that arrowroot, potatoes, and such like bodies, named by him low farinaceous substances, were converted into a species of sugar more difficult of assimilation than the farina of wheat. I very much question whether any proof exists of such differences, and should be disposed to think that, if the absolute amounts of starch or grape-sugar taken in the different amylaceous substance be the same, the effect on the urine would be so also. Direct experiment on this point is, however, still a desideratum.

Fatty and oleaginous substances are usually considered to be advantageous when introduced into a diabetic diet: from my own experience, I am inclined to think very favourably of them when they are easily assimilated by the stomach; and I generally employ cod-liver oil, in order to ensure the patient's taking a sufficiency of these principles. In many cases in which I have administered this substance for some months, and in doses of many ounces per diem, I have estimated its effect on the urine, but cannot say that any very marked diminution of the quantity of this fluid, or of the saccharine matter, occurred. The patients, however, seemed to derive advantage from it, as evidenced by the improvement in their general health; and, after a time, this would be certain to be shown in the condition of the renal secretion.

In the dietetics of diabetes, the liquid portion of the food must not be neglected. Fluids should be taken in as small amounts as the comfort of the patient will allow; but the reduc tion of the quantity should not take place suddenly, as serious symptoms have been known to arise from this cause. The in. gestion of a large quantity of fluid is injurious in more than one way. In the first place, it distends the stomach, and thus produces great discomfort; and, secondly, it seems a well established fact that, the solid diet remaining the same, any increase of liquid taken into the stomach is followed by an augmentation of the saccharine matter excreted by the kidneys-a fact formerly difficult to account for, but now easily explainable on the assumption of the sugar being formed from portal blood; for, when a large amount of liquid is taken, it becomes absorbed by the veins of the portal system, and thus necessarily increases the glycogenic function of the liver by furnishing it with a greater amount of the appropriate pabulum.

Some physicians have been in the habit of advocating the use of alcoholic liquors, from an idea of their acting as respiratory agents. Their exhibition is of very questionable value, and I should be inclined to regard them as rather injurious than otherwise; they doubtless tend to stimulate the liver; and we have seen that, when injected into the portal veins, will themselves produce a saccharine state of the urine.

After a patient has, by means of a properly regulated diet, been brought to a comparatively comfortable state of health and the quantity of urine reduced within very moderate limits, I have reason to believe that a still greater improvement may often be produced by the administration of small doses of opium; and this more especially when, previous to its administration, the patient has exhibited symptoms indicating an un. usually irritable condition of the nervous system. I can bring forward many observations clearly showing this to have been the case; and the occurrences have been too frequent to allow of their being considered merely post hoc effects. In one in- stance under my notice, the numbers obtained by analysis were as follows :-

On diet only, during three days, the averages were-

Quantity of urine, 110 fluidounces.

Specific gravity of ditto, $\mathbf{1 0 3 1} \cdot 9$.

Quantity of sugar, 1679 grains, or $3 \mathrm{oz} .367$ grains.

For seven days, twelve minims of tincture of opium were given three times a day: the amounts were-

Quantity of urine, 105 fluidounces.

Specific gravity of ditto, 1027 .

Quantity of sugar, 1237 grains, or 2 oz. 363 grains.

I have generally found small doses of opium quite sufficient, and that, beyond the amount necessary to allay nervous excitement, a further administration of the drug has produced no beneficial effect; and, even when patients have been brought fully under its influence, the quantity of sugar has not been further diminished. Sometimes I have given it in the form of tincture of opium, sometimes as the solid drug, and not unfrequently in the form of the hydrochlorate or acetate of morphis, but without observing any appreciable difference in the effects produced.

In accordance with the various hypotheses which have been held by different physicians as to the intimate nature of diahetes, a great rariety of drugs have been proposed as remedies, and not $\mathbf{a}$ few have had advocates who have attributed to them curative powers of the highest order. I have felt it my duty, as opportunities have from time to time presented themselves, to give a fair trial to many of these; for, although my anticipations of their value might not have been great, still accurate observations have at least the advantage of determining the real utility, and of preventing the further administration, of unnecessary or even injurious medicines.

Among these various drugs I will first select yeast. Why this substance has been proposed, it is difficult to imagine, except that it possesses the power of converting sugar into carbonic acid and alcohol; and perhaps it might have been supposed to be able to effect this not very desirable change in tbe stomach. Cases are related, I believe, by Dr. Herapath, of the disappearance of sugar from the urine of diabetic patients when this ferment has been administered. In my trials, which, I am sure, have been carefully conducted, I fear I can show no such advantage. It was stated by Mr. McGregor, that, when yeast was given to a diabetic patient, uncomfortable distension of the stomach ensued. I have not observed this effect, although questions have been put for the purpose of ascertaining the point.

In one case-a diabetic man-the yeast was administered for nearly a month, commencing with half-ounce doses three times a day, and then increasing it, first to an ounce three times, and afterwards the same quantity was given six times a day, The urine was not at all improved; it was slightly less in quantity, but higher in specific gravity. No diminution in the amount of sugar occurred.

In another case, also a male, the yeast was given for about a month, in doses of from three to six ounces daily, and with the same result, the daily average of sugar varying from two to three thousand grains, both before and at.the time of taking the yeast. In both the cases the diet remained the same throughout the whole course of the treatment. Yeast appears to me to be almost inert when administered in this disease; it certainly produced no injurious effects : a virtue which cannot be claimed by many of the proposed so-called remedies. I find that a case has been recently published in a French medical journal, showing, by analysis, the inefficiency of yeast in diminution of the sugar in the urine, or in the improvement in the health of diabetic patients.

It would occupy the time of many lectures to attempt giving numbers showing the results which I bave obtained in the use of so many substances. I will therefore group the different drugs in classes, and show the general deduction.

1. Medicines prescribed with the idea that the stomach is in fault in this disease, and therefore that such might remedy the morbid state, in addition to the yeast already alluded to, have consisted of creasote; nitrate of silver; stomachic bitters; nitric, hydrochloric, nitro.hydrochloric, and phosphoric acids; bark, quinine, cinchonine; and the various preparations of iron. No decided effects could be observed. Sometimes, if the appetite was very ravenous, it might be checked by some of these drugs; sometimes, if bad, increased; if anæmia were present, it was relieved: but no decided diminution took place in the amount of urine, and no marked improvement in the character of that secretion.

2. Medicines, the action of which appears to be directed particularly to the nervous centres, have likewise been tried, on 
the supposition that some morbid state of such system might exist. I have already spoken of the use of opium, and considered it of frequent advantage ; but, besides this, belladonna, strychnia, phosphorus, and hydrosulphuret of ammonia, have been prescribed, but with no decided effect, either for good or harm, and certainly no amelioration of the urinary secretion. Dr. Rollo frequently used the hydrosulphuret of ammonia ; but, as he combined its use with a very rigid adherence to an animal diet, it is difficult in his cases to appreciate its real efficacy.

3. The permanganate of potash has been highly extolled, on the supposition that in diabetes there is a deficient oxidation going on; and cases have been related of wonderful cures effected by this agent. When, however, I made trial of this salt, I found that in small doses, such as could be readily tolerated, it produced a most marked increase in the daily amount of sugar; and a patient, who had previously been throwing out between three and four ounces per diem, discharged as much as eight and ten ounces when taking the drug. Dr. Parkes has informed me that in the only case in which he administered the permanganate, the quantity of urine was so notably increased that the drug had to be discontinued. I believe that Dr. Basham has also found a similar effect to follow its employment. I have found, as a rule, that under the influence of many salts, when given in large doses, the urine has always been decidedly increased in quantity; and at the same time the amount of sugar has been augmented; this has been proved in the case of the acetate of potash, the sulphite of soda, phosphate of soda, and many others, to which we shall presently allude.

4. Some years since, at the time when diabetes was regarded as depending on a want of nitrogenised matters in the system, as was supposed to be shown by the deficiency of urea in the urine, the administration of ammonia salts was strongly advocated, and great benefit said to result from their employment. I cannot say that in my experience I have found any difference in the amount of sugar eliminated when patients have been placed, for a few days only, under their influence; but it by no means follows that they are therefore without service, because their continued use may call into activity certain functions, especially that of the skin, which are very imperfectly performed in most cases of this disease.

The idea, however, of highly azotised principles, as urea, ammonia, caffeine, and theine being serviceable, as affording nitrogen to the system, is completely fallacions, and need not at the present day be discussed; some of these drugs, however, may possibly exert other influences, especially on the nervous system. In two diabetic cases I administered a decoction of unroasted coffee in very considerable doses; the only effect observed was restlessness and inability to sleep, but it had no effects upon the excretion of the sugar by the kidneys.

[To be continued.]

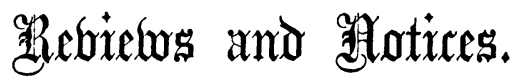

Adumterations Detected; OR Platn Instructions for the Discovery of Frauds in Food and Medicine. By Arthur Hull Hassall, M.D.London; Analyst of "the Lancet Sanitary Commission", etc. pp. 712. London: Longman and Co. 1857.

Some time ago, Dr. Hassaur, whose labours in the exposure of adulterations have gained for him a well deserved reputation, published a large volume, the objects of which were, to demonstrate the existence of extensive adulteration in all the more important articles of daily consumption; to show that adulteration largely affected the pecuniary interests of the consamer and of the revenue; and that it was a question most seriously affecting the public health. In the present smaller work, his object is, "to furnish plain instructions, microsoopical and chemical, embodying the results of extended practice and experience, for the discovery of adulterations in food and medicine". Of the competency of Dr. Hassall for the performance of such a task, it is needless to give proofs, when such ample and extensively known evidence already exists.

Preceding the details relating to the several articles are some excellent introductory remarks, bearing on the subject of adul. teration in general. In the course of these the author discusses the pecuniary, the sanitary, and the moral bearings of adulteration; and gives his opinion as to the remedial measures demanded for the discovery and for the punishment of the fraud. He examines the report of the Parliamentary Committee, and expresses his dissent from several of the recommendations therein contained; while he acknowledges the report to be a highly important document, and the grateful thanks of the public to be due to the members of the Committee for their unremitting labour, during two sessions, in eliciting a mass of most valuable evidence.

The requirements of the case are summed up by the author in the following words :-

" Restrictive measures, beyond those already in existence, calculated either to impede the freedom of trade or to curtail the liberty of the trader or the subject, are not required for the suppression of adulteration. What is needed is an effective scientific organisation for the discovery of adulteration, and an adequate punishment of the offence."

The work is enriched with two hundred and twenty-five woodcuts, illustrative of the microscopical appearances of the various articles described; and is rendered easy of reference by a copious index. Further, it is of portable size, and is published at a moderate price. When to these recommendations is added the fact of its being a sound practical treatise, it is evident that it must soon command an extensive circulation.

Cuinical Lectures on the Diseases of Women and Chmoren. By Gunning S. Bedfond, A.M., M.D., Professor of Obstetrics, the Diseases of Women and Children, and Clinical Midwifery, in the University of New York. Fourth edition, carefully revised and enlarged. pp. 602. New York: S. S. and W. Wood. London: Sampson Low, Sons, and Co. 1856. On the Diseases of Women: including those of Pregnancy and Childbed. By Fleetwood Churchill, M.D.T.C.D., M.R.I.A., Vice-President and Fellow of the King and Queen's College of Physicians in Ireland. F'ourth edition. pp. 827. Dublin : Fannin and Co. London: Longman and Co. 1857 .

DR. BEDFORD's work consists of thirty-one lectures, each containing the histories of and remarks on several cases, thrown together apparently without the least attempt at classification, but published just as they were delivered before his class, as the cases were presented from day to day. The style of the author is very graphic, and he adopts the system of recording actual dialogues with his patients-a plan which, although it may seem peculiar, is often a most efficient mode of impressing a fact on the mind of the hearer or of the reader. The practical merits of the work are sufficiently shown, if by nothing else, by the fact of its having reached a fourth edition in less than two years: and not only does the book prove the author to be a sound physician and an excellent clinical teacher, but it gives evidence of an extensive acquaintance on his part with the literature of his subject on this side of the Atlantic.

Dr. FleEtwood Churchinl's work consists of three Books; of which Book $\mathrm{r}$ treats of Diseases of the External and of the Internal Genital Organs; Book In of Diseases of Pregnancy; and Book III of Diseases of Childbed. This edition has been enlarged and improved, and is for the most part brought down to the present state of knowledge. We notice, however, that the author does not seem aware that Dr. Trask, of whose valuable statistical memoir on Rupture of the Uterus, published in the American Journal of the Medical Sciences in 1848, he speaks with high commendation, published a completion of the essay in the same periodical for July 1856: an abstract of which was given in the Association Medical Jounnal on October 4th. This we mention, not to disparage the general merit of Dr. Churchill's work but merely to point out an oversight, which no doubt will remedied before long, when another edition is called for. 